\title{
Exploring Mobile Ad Formats to Increase Brand Recollection and Enhance User Experience
}

\author{
Aku Visuri, Simo Hosio, Denzil Ferreira \\ Center for Ubiquitous Computing \\ University of Oulu, Finland \\ \{firstname.lastname\}@oulu.fi
}

\begin{abstract}
Digital marketing is increasingly moving from desktop (e.g., browser) to mobile environments (e.g., within mobile applications). The means for delivering ads however, remains largely unchanged: banners and videos. In this work, we explore transforming ad delivery methods to the mobile environment while mitigating issues causing frustration and distractions to the users, evident in both web and mobile marketing. We demonstrate that softly enforcing interaction with the ad - with minimal usable screen space reduction - can improve user's attitude towards mobile advertising. Brand recollection is also influenced via increased interactions with the ad delivery method.
\end{abstract}

\section{Author Keywords}

Smartphone; advertising; interfaces.

\section{ACM Classification Keywords}

H.5.m. Information interfaces and presentation (e.g., HCI): Miscellaneous;

\section{INTRODUCTION}

Mobile advertising has experienced significant changes over the years, transforming from non-interactive text messages to highly personalised and context-aware instances [9]. However, mobile advertisements are generally ignored by users [3] or can cause users to feel distracted or irritated. Entertainment and enjoyment have both been identified as key metrics in analysing the effectiveness of mobile advertisements and peoples' attitude towards the advertisements [14,18]. Same studies also report how the advertisement delivery method should consider the user's ability to interact with her mobile device. Presenting the advertisement in proper context can also generate utilitarian benefits for the user [10].

Permission to make digital or hard copies of all or part of this work for personal or classroom use is granted without fee provided that copies are not made or distributed for profit or commercial advantage and that copies bear this notice and the full citation on the first page. Copyrights for components of this work owned by others than the author(s) must be honored. Abstracting with credit is permitted. To copy otherwise, or republish, to post on servers or to redistribute to lists, requires prior specific permission and/or a fee. Request permissions from Permissions@acm.org.

MUM 2017, November 26-29, 2017, Stuttgart, Germany

(C) 2017 Copyright is held by the owner/author(s). Publication rights licensed to ACM.

ACM ISBN 978-1-4503-5378-6/17/11 \$15.00

https://doi.org/10.1145/3152832.3152834
A frequently used form for delivering mobile ads are banners - shown within the application's content, or on the top or bottom sections of the screen. However, a trait learned by users on desktop computers has also transformed to smartphone use, and causes users' to habitually dismiss the contents of such ads. The concept of 'banner blindness' [3] was coined in 1998 and refers to people's ability to scan web pages swiftly while ignoring banner content, to the extent that they have a hard time recollecting the banner's contents [4]. Yet, mobile applications for the most part rely on showing advertisements in a very similar fashion.

In this paper, we explore novel mobile ad delivery methods for improving: 1) ad content recall; and 2) user's interaction frequency with mobile advertisements. More specifically, we use a floating icon with minimal information about the ad to deploy mobile advertisements during active smartphone use, and to prompt the user to interact with the ad to gain more information about the advertised product or service. We contrast our approach against the traditional method of delivering banner ads in terms and obtain both qualitative (interviews) and quantitative (smartphone sensor data) feedback from the participants. We organised workshops where ten participants performed predefined smartphone use cases, before and after which they were surveyed and interviewed regarding ad recall and opinions on the advertisement techniques in general. We find that users' attitudes towards ads and brand recollection can be influenced by designing ads that enforce soft interactions but do not require excess screen space.

\section{RELATED WORK}

Advertising is an audio or visual form of marketing communication and employs an openly sponsored message to promote or sell a product, service, or an idea [16]. The purpose of advertising is to encourage the consumption of products or services via branding - increasing user knowledge of brands by associating the product name or image with qualities in the mind of the consumer. Branding is a rather simple way of marketing because it does not require any specific context to be effective. Another approach is direct response advertising, which aims to directly sell products, but it is often difficult to correctly predict user preferences, especially in mobile advertising, compared to methods like situated marketing (e.g., within stores, or during TV shows) [16]. SmartAds is an approach 
by Nath et al. [15], aiming to reduce amount of irrelevant ads presented to the user. Bart et al. [2] analysed which types of products or advertising methods are best suited for mobile advertising, and found that smart devices are a great medium for increasing brand recollection by showing repeated (yet relevant) information the user's recall of previously stored information is easily triggered.

Digital marketing is increasingly directed to consumers via mobile technologies. The projected share of digital marketing on mobile devices for 2017 (in a 2016 report) was $33.3 \%$, and is projected to increase rapidly [11]. Numerous difficulties impact digital marketing via smartphones, and consumers generally have a negative bias towards mobile advertising, especially if they have not consented to it [17] - which is the case for many applications, i.e., applications do not specifically ask for user's permission to show ads, merely inform of the present ads. Numerous studies have analysed the core effects of models like the Technology Acceptance Model (TAM) [8] and more fine-grained models investigating e.g. emotional attachment to our smartphones [19], or the interactive aspect of modern smartphones [12], as a way to assess acceptance towards mobile advertisements: "interactivity on mobile devices is positively related to consumer commitment and dispersion of social networks for product and service information".

Other aspects influencing our acceptance of smartphone ads are, e.g., behavioural intention (to buy) certain products, the
Unified Theory of Acceptance and User of Technology (UTAUT), and perceived enjoyment and mobile skillfulness [18]. These share the rationale that interactive or "playful" ads connect well to the user's hedonistic utilitarianism, increasing ad acceptance. Similar results are reported in [14]: entertainment, irritation, and usefulness are key drivers of teenagers' attitudes towards mobile advertising, and perceived usefulness reduces irritation.

While it is technically trivial to measure e.g. how frequently people click banner ads or watch through video ads, the statistics are largely a trade secret. In general, the clickthrough rates can be considered very low, however. Prestige Marketing report $2.1 \%$ of banner ads resulting in clicks [13].

Older people are more likely to click on ads, and most frequent reasons for not selecting to click are fears of viruses and spam, and the ads being distractive. Kushal et al. [7] created a contextual model that can increase clickthrough rates, but note that is still difficult to increase CTRs if the initial CTR is low. Cho et al. [6] note that for banner ads, the intrinsic level of interest in the product, the delivery method's fit to the product, self-perceived attitude towards the delivery method, and overall attitude towards online advertising are key factors in determining CTRs.

For video ads, Mirriad Advertising claims that up to $90 \%$ of ad videos are skipped [1] and note that younger people tend to prefer less distractive ad styles, and dislike traditional style of advertising, such as video commercials. It has also

\begin{tabular}{|c|c|c|c|c|}
\hline Ad type & Removal Method & Effect on Screen Space & $\begin{array}{c}\text { Level of } \\
\text { Distraction }\end{array}$ & $\begin{array}{c}\text { Impact of Repetition } \\
\text { on Distraction }\end{array}$ \\
\hline $\begin{array}{r}\text { Banner (fixed) } \\
\text { e.g. full screen apps, } \\
\text { games }\end{array}$ & $\begin{array}{l}\text { Not available } \\
\text { (usually) }\end{array}$ & $\begin{array}{l}\text { Medium (often overlaid on top } \\
\text { of interface elements) }\end{array}$ & Medium & Medium \\
\hline $\begin{array}{r}\text { Banner (dynamic) } \\
\text { e.g. list views, Gmail }\end{array}$ & $\begin{array}{l}\text { Not available } \\
\text { (usually) }\end{array}$ & $\begin{array}{l}\text { Small (reduces amount of } \\
\text { information but does not } \\
\text { overlap with interface) }\end{array}$ & Low & Low \\
\hline $\begin{array}{r}\text { Video (full screen) } \\
\text { e.g. Youtube }\end{array}$ & $\begin{array}{l}\text { Not available, or } \\
\text { 'skip after } \mathrm{x} \\
\text { seconds' }\end{array}$ & High & High & High \\
\hline $\begin{array}{r}\text { Video (embedded) } \\
\text { e.g. webpages }\end{array}$ & $\begin{array}{l}\text { Not available, } \\
\text { pause, or 'skip after } \\
\text { x seconds }\end{array}$ & High & High & Medium \\
\hline $\begin{array}{r}\text { Image (full screen) } \\
\text { e.g. webpages, popups }\end{array}$ & $\begin{array}{l}\text { Click designated } \\
\text { location to close }\end{array}$ & High & High & High \\
\hline $\begin{array}{r}\text { Audio } \\
\text { e.g. Spotify }\end{array}$ & $\begin{array}{l}\text { Not available, or } \\
\text { 'skip after } \mathrm{x} \\
\text { seconds' }\end{array}$ & None & Low & Medium \\
\hline In-app & $\begin{array}{l}\text { Usually not } \\
\text { available }\end{array}$ & Varies & Varies & Varies \\
\hline Proposed Icon & Drag to remove & Small & Low & Low-Medium \\
\hline
\end{tabular}

Table 1. Different types of mobile ad delivery methods, with corresponding variables. 
been noted regarding more intrusive methods, such as alert dialogs, that the user's initial reaction to any alert dialog is to click on the cancel button [5] - which makes these types of delivery methods extremely ineffective as the user's focus shift completely from any information content presented to him.

\section{MOBILE AD DELIVERY METHODS}

Mobile ads generally use a combination of text and images, video, or audio to deliver a promotional message to the user. Different methods take up different amounts of screen space, have different interaction methods (basically ways to remove the ads), and cause different levels of distraction and distress to the user. Finally, some delivery methods are more easily repeated without causing significant amount of distraction.

We summarise the main ad types utilised in mobile advertising in Table 1, alongside with the interaction levels and our expert assessment of the different ways the delivery method can influence user experience. We selected the influencing variables based on most commonly seen negative traits of mobile (or desktop) advertising methods. In Figure 1, we have included examples of banner ads, video ads, and interfaces showing combinations of both. We have also included in-app ads as a group, such as in-app purchases or other products from the same publisher. The interfaces of these apps, however, vary a lot so it is difficult to generalise their impact on the listed variables.

Figure 1a shows an example of a static banner ad that is overlaid on top of the map interface, Figure $1 \mathrm{~b}$ and Figure 1d show examples of dynamic banner ads, showing as members of existing list views, and Figure 1c shows an example of a large banner ad. The interaction method with the ads is somewhat evident in most of the examples, with clear 'install' or 'skip' options, except for Figure 1c where
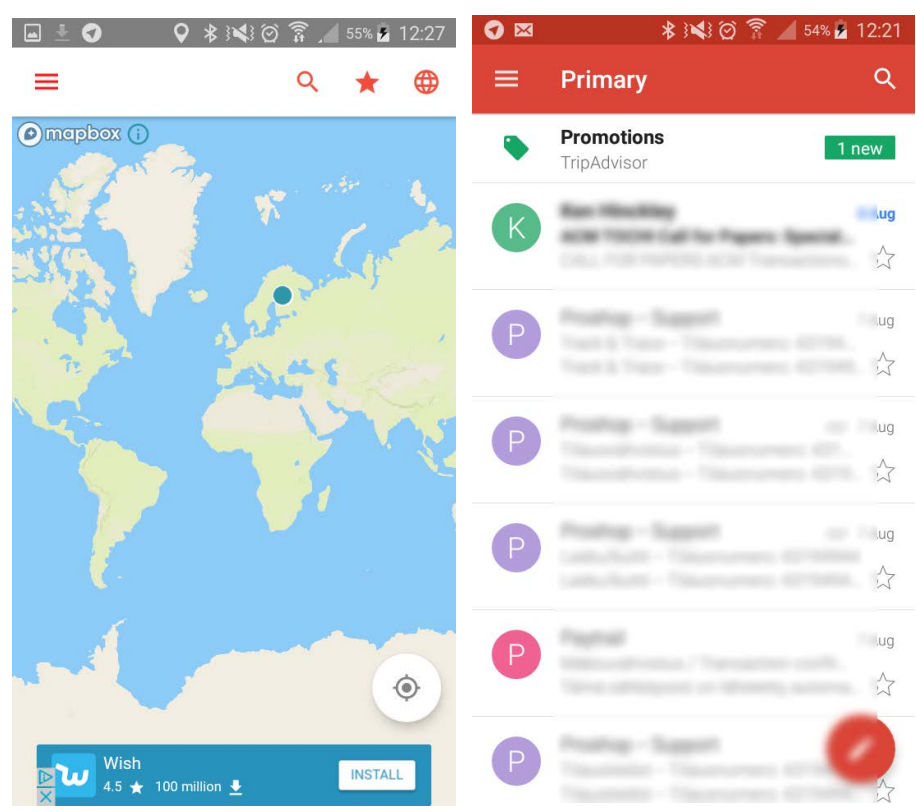

it is not directly clear what happens if the user clicks any other area of the ad except for the 'install now' text. Example Figure 1d is the only one with a clear remove button (the cross), while expert users know that figure-b can be swiped away. Examples Figure 1a and Figure 1c do not have any removal options. The issues commonly associated with the ad overlaying on the interface are not directly evident in Figure 1a, but when considering another full screen

applications - such as mobile games which require more complex interfaces - the issue of interface elements being hidden beneath ads becomes evident.

Main causes for irritation towards ad delivery methods are reduced interface spaces (e.g., banner ads), ads that overlay the interface and can have potentially hidden closing mechanisms (e.g., popups and dialogs), and videos that play automatically or are not skippable. These types of ad delivery methods share common trait as they reduce or alter the available interface space and are hard to hide or get rid of. In our experiment, we designed an interface that aims to mitigate the issues, listed as variables in Table 1, while still being able to present the user with adequate information. The expected impact of our evaluated delivery method according to the listed variables is also included in the table. Next, we describe our experiment and the results and analysis of the experiment.

\section{EXPERIMENTAL SETUP}

We evaluated our approach using an android application that delivers advertisements using two distinct delivery methods. We presented advertisements from 20 different brands to ten participants, each in an hour-long workshop, and conducted interviews with each participant at the beginning and end of the workshop.

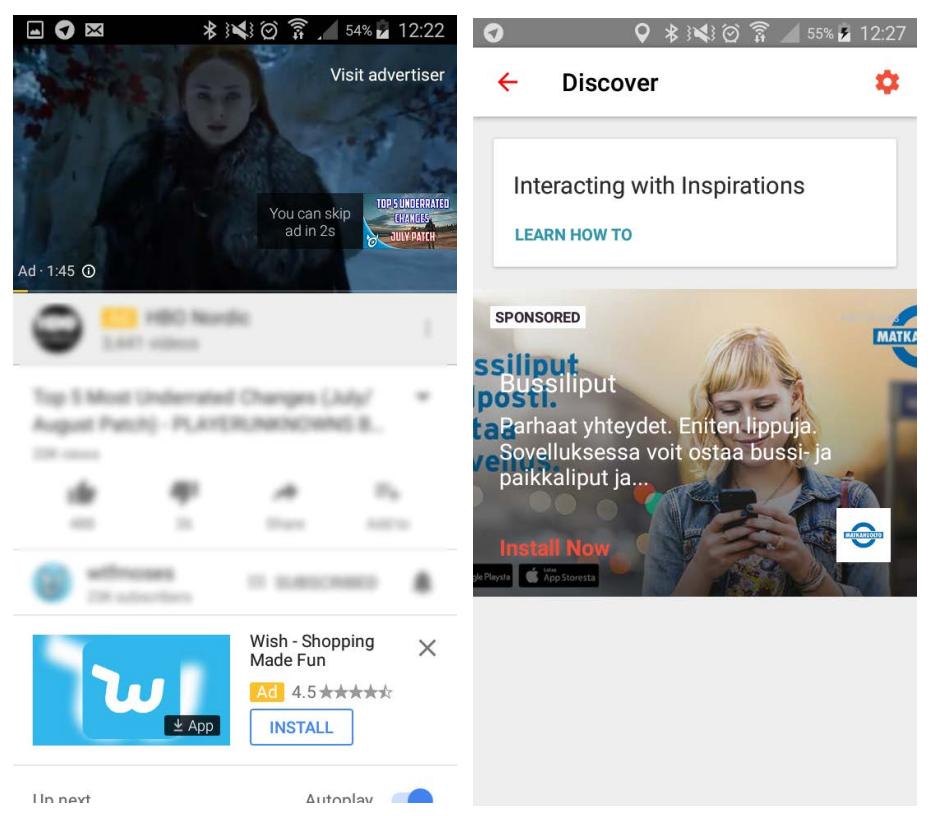

Figure 1. Examples of mobile ad delivery methods: a) Static banner, b) dynamic banner,

c) video and dynamic banner, d) large static banner. 

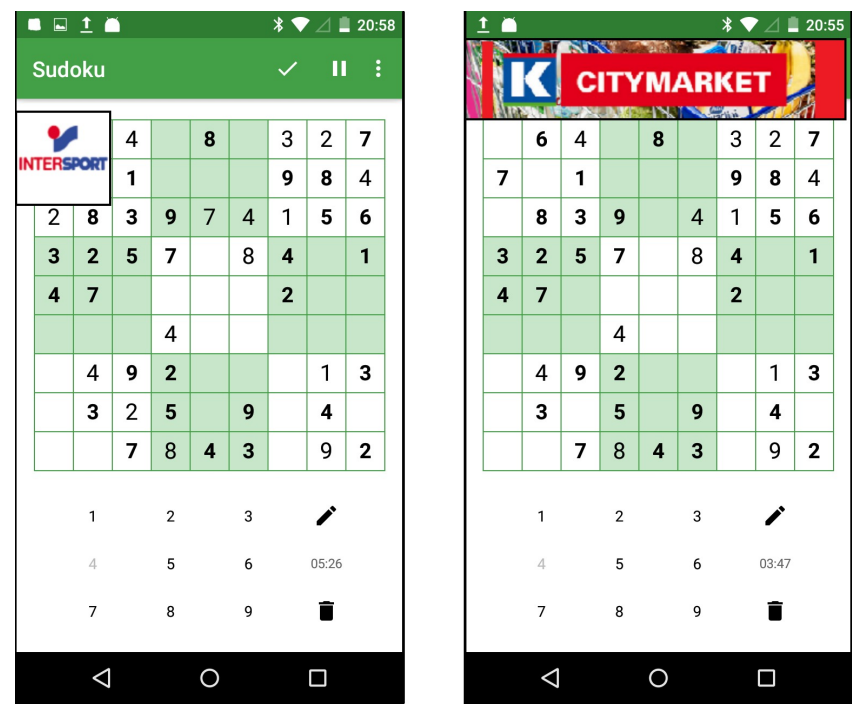

Figure 2. Examples of the icon and banner ad delivery methods, overlaid on the Sudoku application used in the experiment.

\section{Application}

We designed an android application to deliver advertisements using two different mechanisms - traditional banner ad and a small movable icon. Both were implemented using the Android Floating Action Button widget, and examples of both can be seen in Figure 2.

- The banner advertisements are shown on the top of the screen, spanning the total width of the screen. The banner is static and displays the brand name and/or logo and can be clicked to display an alert window, similar to the icon mechanism.

- The movable ad icon is displayed on top of any interface, can be moved around, and removed by dragging the icon the bottom of the screen - just like the Facebook Messenger "chat heads." The icon is always initially placed on the top left corner of the screen. The icon displays a brand icon and/or name when in icon-mode, and when clicked, displays an alert window with more detailed information about the brand and advertised product or service.

We use a game of Sudoku as the active application in our experiment, as the game's interface contains interactive elements throughout and is symmetrical in nature. We selected a total of 20 businesses with ongoing online advertising campaigns. The brands were chosen to show diversity in both the brand type (e.g. news, restaurants, grocery stores, travel services) and the presentation style (e.g. font choice and color). We chose to present real-world ads to increase the authenticity in the experiment.

\section{Procedure}

Before each interview session, we ensured that the smartphone used for tests (LG Nexus 5 with 4.95" display) was functioning correctly, and that the test setup was reset appropriately. Each participant was individually invited to a quiet place, and the test was conducted with two researchers present during the test - one to help with the experiment and the other one to unobtrusively observe the participant and $\log$ any interesting interactions. We explain to the participant that the purpose of our experiment is to evaluate two different mechanisms for delivering mobile advertisements and explain the two different delivery mechanisms.

We collect demographic information of each participant (age, sex), as well as information about their daily smartphone use (what do they use smartphone for, and how frequently they use smartphone applications). Next, we collect information about their experiences with mobile ads with the following set of questions:

- What types of ads have you experienced on a mobile device?

- Which of the following mobile ad presentation mechanisms have you observed on mobile devices? (Banner, full screen, application, voice, video, other)

- Do mobile ads influence your application use? How? If they are disruptive, which types are considered disruptive?

- Do mobile ads elicit any emotions?

- How often do you read or interact with mobile ads?

- How often do mobile ads elicit interest in you?

- What types of mobile ads elicit most interest in you?

- What are your general thoughts about ads in mobile applications?

Next, the participant is asked to play four five-minute long rounds of Sudoku. The participant is instructed to play at a pace of their choosing, and that the goal of the experiment is not to finish a round - this was highlighted to the participant to ensure they will not experience all ads as disrupting to them rushing to finish the round. During each five-minute round of play, ads are delivered to the user at varying intervals of 30-60 seconds, leading to each participant seeing minimum of five and up to ten unique ads during each round. There is a brief period (5-20 seconds) of no ads being shown before each new ad, for both methods, and the ad is automatically removed in the beginning of this period if it was not removed explicitly by the participant.

The order of the shown ads is randomised at the beginning of each round. We alternate and balance the delivery method by selecting five participants that begin with the banner delivery method (banner-icon-banner-icon), and five participants that begin with the icon delivery method (iconbanner-icon-banner). We encourage the participants to not completely concentrate in playing the game during the experiment, and to engage in light conversation with the researchers during the game, comment on the game, or ask 
or answer short questions when applicable. We considered this due to participants potentially experiencing any ads more distracting if they were overly focused on their task. Primarily, we wished the participants to feel as comfortable as possible during the experiment to capture a more realistic smartphone use experience. During the experiment, we observe the participant as they play the Sudoku game and interact (or opt-out) with the advertisements during the game.

After the experiment, we collect information about the participants' experience with each delivery method, and with the advertisements. First, we ask each participant which advertisements (brands) they remember seeing during the experiment, and give them a few minutes to think back. As explained previously, the ads were selected from local businesses in a way that ensured that all participants were previously familiar with the brand names to reduce participant's recollection bias towards previously known brands. Next, we ask the following set of questions regarding the usability of both delivery methods:

- Did the icon delivery method influence the usability of the application?

- Did the banner delivery method influence the usability of the application?

- What is your general opinion on the icon delivery method?

- What is your general opinion on the banner delivery method?

- Which delivery method would you prefer?

- What aspects of mobile ads influence their irritative nature?

- What aspects of mobile ads influence content delivery?

- Other general comments?

The whole experimental procedure took approximately 6070 minutes per participant.

\section{Data Collection}

We collected data from the device during the test to investigate how the different ad delivery method affects people clicking the ad and recollecting their content. Further, we logged any additional interactions with the icon type ad (i.e. moving the icon around on the screen, manually dismissing it).

For each ad that was triggered to the users we logged the delivery method and ad ID (brand name). We measured the number of interactions (clicks and touches, associated to moving the icon) with the ad, where the icon was interacted with (screen coordinates), the duration it was displayed for, whether it was removed by the participant or the system (each ad automatically removed after 30 seconds), and whether the participant reported seeing the ad after the experiment. We summarise each participants' interview information in terms of prior mobile ad knowledge and user's potential negative bias towards ads, and store it along each presented ad for use in data analysis.

\section{Participants}

We recruited ten participants for our experiment, of which nine were in their mid-20s (aged 22-27, 3 female, 6 male), and one older (aged 58) female participant (P5). Majority of participants $(\mathrm{N}=9)$ had good prior knowledge score (score of 4 or 5 , rated 1-6 according to how many ad delivery methods the participant was previously familiar with), and P9 had a score of six. Two users (P2 and P4) had high prior negative bias (two on a scale of 0-2) towards mobile ads, while three users (P3, P7, and P9) had no negative bias (value of zero).

\section{RESULTS}

First, we collected prior experiences with mobile ads from our participants to see how their potential negative experiences match the literature. We then compare how participants interacted with the two delivery methods (banners, icons) during the experiment, and how often participants remembered seeing a particular ad, based on their own recollection after the evaluation. We use the delivery method, displayed ad, participants' individual differences in age, sex, knowledge of mobile advertisement mechanisms, and potential prior negative bias towards advertisements as independent variables to study their influence on recall and number of interactions (i.e., times the ad was touched or clicked). Finally, we report the qualitative feedback collected in the sessions.

\section{Prior Experiences}

Overall, the participants' past experiences with mobile ads were fairly neutral $(\mathrm{M}=0.9$ prior negative bias on a scale of $0-2$ ), but participants frequently noted having negative experiences with ads. Comments exemplifying users rarely receiving relevant ads include such as

$$
\begin{aligned}
& \text { "I receive ads from distant locations very often" (P5), } \\
& \text { "Maybe once every few weeks" (P7). }
\end{aligned}
$$

Further, ads were perceived to negatively influence device use or being otherwise annoying or even anger-provoking:

"I dislike when ads take up screen space” (P4),

"I can manage ads when I know a certain app contains them, but hate being surprised" (P7).

Participants also reported being able to swiftly generate an opinion on an irrelevant ad and appreciate relevant ads:

"If I'm not interested after the first second upon seeing an ad, I will generally ignore it or get rid of it" (P5),

"I'm most interested in ads that are based on my search history” (P2).

To summarise, the participants generally disliked when ads reduce their user experience by altering the user interface, and prefer to see relevant ads. 


\section{Interactions}

We investigate the effect of the ad delivery method (type) to the amount of click/touch interactions and whether the participant remembered seeing an ad or not during the experiment. A total of 197 ads were shown to the participants (each participant saw each ad at least once, except for P1, P3, and P10 who saw nine unique icon ads), and participants interacted (via touching) with the ads an average of 26.6 times (1.33 per ad).

The banner ads were not clicked at all during the experiment $(\mathrm{N}=155)$, whereas $11.7 \%$ (13 of 111$)$ of the icons were clicked. While the banner ad is certainly larger than the icon, the information presented by both mediums in their primary state is the same. Users can potentially open the ad via misclick in both delivery methods, but we did not observe this behaviour during the experiment. The ads were clicked by six different users (P1, P2, P3, P4, P9, and P10), and curiously only $\mathrm{P} 4$ and $\mathrm{P} 5$ remembered seeing the ads they had clicked. The clicked and remembered ads were not biased towards ads shown either in the beginning or the end of the experiment, accordingly to the display order of the ads. None of the clicked ads were removed by the user, and five were interacted with by moving the ad around the screen while it was displayed. Using ChiSquared we can observe that differences in click ratios are impacted by the delivery method $\left(\mathrm{x}^{2}=16.64, \mathrm{p}<.05\right)$, whereas user recollection was not $(\mathrm{p}=.53)$.

For interactions with the icons, we measure the duration each presented icon was visible and how many times users touched the corresponding icon. The average duration each icon was visible for was 27.6 seconds (47.0s for banner ads), and icons were touched an average of 1.26 times ( $p<$ $.05)$. Median touch count was one, indicating most icons were simply either removed by the user $(53.2 \%, \mathrm{~N}=59)$ or moved once and then ignored until removed automatically $(46.8 \%, \mathrm{~N}=52)$. Using Chi-Squared, we uncover an effect with the ads visibility duration and number of touches, but there is no direct correlation with the variables - which could be a somewhat expected result. Instead, the icons seem to attract the participant's attention during the first 25

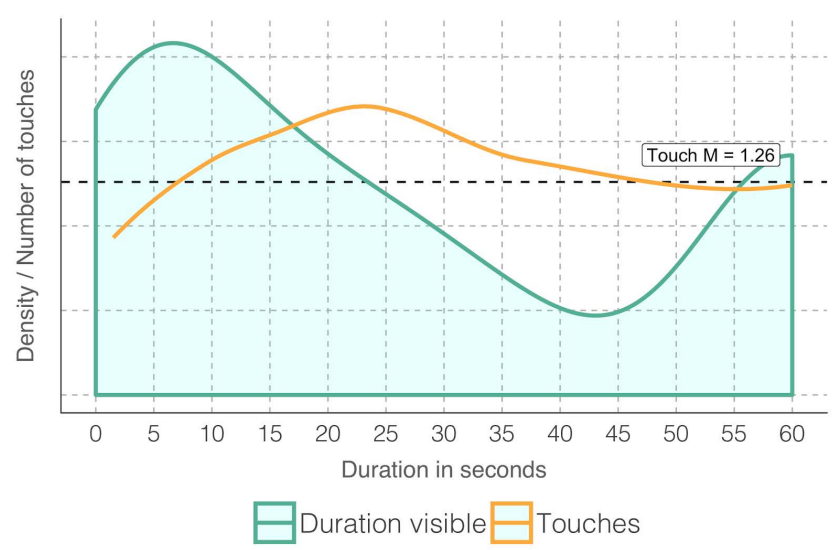

Figure 4. Distribution of visibility duration for each icon, and the number of touches for each icon.

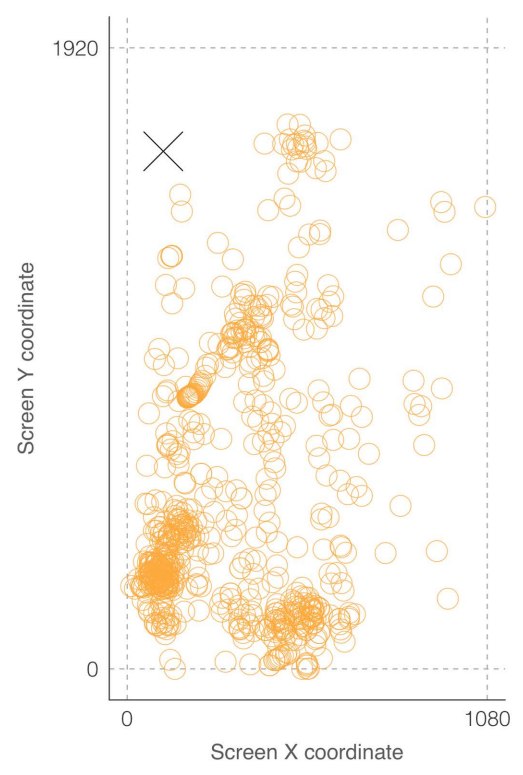

Figure 3. Distribution of icon touches per screen coordinates of actions end point. $X$ denotes the starting point of the icon.

seconds, after which the number of touches start to diminish. Majority of the icons are short-lived, as $40 \%$ for 11.8 seconds or less and $50 \%$ of icons are only visible for 22.9 seconds or less. This indicated that majority of the user removed icons are removed by the user rather swiftly. The frequency of visible duration of each icon and the distribution of touches are visualised below in Figure 4.

Lastly, we logged where on the screen the icons are interacted with by touching and moving them around. The interactions are visualised in Figure 3. As the icon was always initially placed on the top left, this elicited the participants to generally interact with the icon on the left side of the screen. As the interface of the sudoku game is symmetrical, the occasions where the icon is in the way (hiding away some of the numbers on the gaming area) are symmetrical regardless of the icon's location. Thus, when the user required to see the top-left corner of the game, they habitually moved the icon down, or in some occasions slightly to the right. The participants also often removed the icon by simply dragging it to the lower-left corner, instead of the actual 'remove icon' located lower-middle.

\section{Observations}

During the experiment, we observed the participants to remove any false positives (accidental clicks) and to capture more subjective information regarding their experience. As previously reported, the banner ads were not clicked during the experiment, and we could also verify during the experiment that the participants did not open any of the icons ads via misclicking. Rather, P8 noted removing two of the icons by accident in the beginning of his first round of play, saying that he just wanted to put the icon "on hold". All participants also seemed to enjoy moving the icons around during the game, either to pass the time or give 
them a small break to think about their possible moves (as revealed later during the interviews).

We postured that the interactability with the icon, alongside the curiosity prompted by the lack of information presented by the small icon, could be the main source of motivation to click the icon. The participants seemed to experience that the larger banner ad offered sufficient amount of information, while they were curious to learn more from the icon - although the initial information (brand name and logo, with no detailed information about the advertised product) was the same for both delivery methods.

\section{Usability of the Icon Delivery Method}

The participants were asked about the impact of the icon interface on the 1) usability of the application, and 2) the overall user experience with the mobile ad. They reported that the icon influenced their gaming experience by hiding a portion of the puzzle:

"It was a bit difficult because the icon would sometimes hide some of the numbers" (P2)

and that it would likely be experienced disrupting if an application would constantly send such icons:

"I would probably be annoyed if a game would constantly bombard me with them" (P3).

Regardless, seven participants (P1, P4, P5, P6, P7, P8, and P9) thought the icon mechanism was novel, smart, interesting, and that the ability to swiftly remove an ad was a great feature:

"Clever way to advertise, as long as they aren't presented constantly. Not that disruptive and easy to remove if I'm not interested in the ad" (P1),

"I thought it was fun - the icon was small and you could play around with it, sort of like a game. And it was easy to remove" (P9).

Two participants (P2 and P10) noted that the icon mechanism was confusing and that they would rather look at a static banner. P8 also noted that due to small size the icon itself can offer very limited amount of information.

\section{Preference for Banners or Icons?}

Finally, we inquired the participants about their experiences with the banner mechanism, and comments and arguments for which method they would prefer. The main finding regarding the banner mechanism was that it is considered a very traditional mechanism and that the participants were highly prone to banner blindness [3]:

"It's very bland and boring. I'm so used to seeing banner ads I tend to just ignore them." (P4).

This was also indicated - but not verified - by the statistics on remembering seen ads based on delivery type. The participants commented that they find the banners annoying as there is often no way to get rid of them and they take up precious space on the interface:
"The banners were quite irritating because there was no way to get rid of them and the application felt very cluttered",

"Although the banner was not overlapping the gaming interface it still felt like it was in the way" (P1) - and that they are either afraid to click them, not knowing what will happen - e.g. a webpage launches or a full screen ad appears:

"I am afraid to click them, because they might take me to a weird webpage or force me to download something accidentally" (P7)

or that they sometimes click them by accident due to the large size or location:

"When apps contain banners located on the bottom of the screen I sometimes click them by accident" (P4).

Being able to get rid of an ad when needed was considered a great quality, and was highlighted by several participants as the redeeming quality of the icon mechanism. The mechanism for hiding the ad was also not hidden and the required action was experienced as simple:

"I liked how it was very fast to remove unwanted ads" (P6).

Another upside was that it was considered subtle:

"The icons did not make the app look cheap, which sometimes happens with tacky banners" (P5).

The experienced downsides of the icon mechanism were small size and the fact that since they could be removed they could be easily forgotten:

"The icon is quite small, and if I do not already know the brand it is very hard to decipher it (e.g. the name) from the icon" (P3),

"I just focused on removing the icon so I think I just forgot them all” (P3).

Interestingly, P3 happened to still remember seeing more $(40 \%, \mathrm{~N}=4)$ icon ads than banner ads $(20 \%, \mathrm{~N}=2)$.

Ultimately, five of the ten participants preferred the icon mechanism, three preferred banners, and two were undecided, both stating that as long as the icon would not hinder the overall use of the interface, it would be a better option.

\section{DISCUSSION}

The goal of our experiment was an attempt to mitigate the issues in mobile ads that cause frustrating user experiences or cause the ad delivery methods to be ineffective, due to e.g. users ignoring the ad content. While our experiment does not directly approach the overarching marketing themes such as brand recollection or increasing product sales - as these topics are not within the scope of the experiment and thoroughly researcher in other fields - the results have an indirect impact by highlighting how the overall attitudes towards mobile advertising can be 
influenced. Similar to other studies [2,15], our participants highlighted how ad relevance is key in determining the acceptance towards mobile ads.

To start off, smartphones are a different media than e.g. desktop web browsers, where the screen space is relatively large - the resolution could be the same but the physical constraints are vastly different, i.e. a 4.5" screen on a smartphone versus a 27 " screen on a desktop computer. Yet, developers of ad delivery methods assess that similar absolute screen space can be spent on showing ads - i.e. the physical $(\mathrm{cm} \times \mathrm{cm})$ size of the ad can be similar. But when you think about the constraints of the already small screen, taking up greatly increased relative space for an ad can and has been shown to be experienced extremely irritating by smartphone users. It is understandable that an ad cannot be too small, or it could be incomprehensive and unreadable, but other considerations should be considered to reduce the required screen space. Ads taking up screen space, being difficult to interact with, and users being "surprised" by ads were key aspects brought up by our participants. Overall, our participants seemed to find similar issues in mobile advertising, to what has been previously reported, and were a fitting target audience to evaluate whether these issues could be mitigated.

Anecdotally it could be considered that exposure (visibility duration in our experiment variables) to an ad would increase user's awareness of the ad, and make it more likely for the user to notice and recall the ad, thus leading to increased brand awareness or product purchase (depending on the goal of the ad). Thus, offering the user an option to remove an ad seems counterintuitive (which most banner ads in particular do not offer). We did not find such correlation, however, and we even revealed a case of P3 implying such impact ("I just focused on removing the icon so I think I just forgot them all") - but in fact being more likely to remember seeing the icon ads which she interacted with and which were presented to her for a shorter duration.

The participants remembered seeing more of the icon ads $(33.3 \%>29.0 \%)$, even though they were generally visible for shorter duration $(27.6 \mathrm{~s}<47.0 \mathrm{~s})$. One important finding regarding duration was highlighted for the icon method, as icons that there quickly removed $(<10 \mathrm{~s})$ were less likely to be remembered $(6.6 \%<46.6 \%)$ by the users than the icons that were visible between 10 and 30 seconds. Participants were also more likely to remember ads visible for 10-30 seconds than the ones that were generally left for the system to remove (visible for more than 30 seconds). Icon touches also follow this same style of trend, as shown in Figure 4. Seems like having exposure to an ad and interacting with it (even if the interaction is enforced via the required removal) can potentially increase user's brand recollection and thus, brand awareness. Prior knowledge and preference of a brand can also influence how interesting the ad is considered, and how likely the participant was to remember seeing it. This is a known limitation of our study setting and something we sadly cannot control for.
Key factor is the user being aware of the ad, and this can be softly enforced by a) minimising the negative influences like reduced screen space and b) allowing the interaction in an interesting way, opposed to popular approaches like the "click $X$ to close", or even the "find X to close" in some cases. Additionally, allowing the user to freely continue the task he was performing without large interference makes the ad become less intrusive. These are the aspects we took into consideration when designing the icon interface as an alternative, and our experiment showcases success in mitigating these factors.

Lastly, when looking back to Table 1 and the assumptions made for our icon delivery method, we can verify that the results provided in our experiment reassure of these assumptions. Our participants repeatedly noted that the icons did not take up excessive screen space and the icons could be swiftly removed - and that the removal method was intuitive and simple enough. This is sometimes not the case for mobile and desktop advertisements, which occasionally require the user to go through unnecessarily lengths to get rid of unwanted content and interruptions. While any unwanted information can be seen as distractive, smaller ad delivery methods can reduce the level of disruption - as expected - while still retaining adequate amount of information to elicit interest. And, similar to any advertising method, overburdening the user with constant flow of distractions is undesired - put in to words by P1 and P3, both noting that the experience with the icons was positive, as long as they were not presented in excess.

We can conclude from our experiment that there is room for mobile advertising mechanisms to evolve, and that the user experience should be strongly taken into consideration when designing ad delivery methods. This has strong impact on general receptiveness to advertisements, and thus on the end results of advertising campaigns. The traditional direct translation of delivery methods from desktop environments to mobile is sub-optimal, and the mobile requires novel approaches.

\section{Limitations}

In our experiment, we displayed a fixed pre-generated array of ads to the participants, and users might have biases towards some of the brands, resulting in them being more likely to recollect seeing a brand they are interested in. However, we controlled for this by carefully selecting the brands in a way that ensures that all participants should be familiar with all displayed brands. Additionally, separate brands were delivered for each method, as this was necessary to appropriately differentiate between the delivery methods.

\section{CONCLUSION}

We conducted an experiment to evaluate means to mitigate common issues associated with mobile ad delivery methods. We investigate impact on attitudes towards mobile ads and delivery methods, and how users interact with mobile ads. We designed a movable icon that takes up reduced amounts of screen space (compared to generally 
used methods), and can be moved around in case it overlays a critical portion of the screen. The icon an also be effortlessly removed if the user considers the presented ad irrelevant or shown at inappropriate time, which can often be the case.

Our results highlight that simply exposing users to ads, preferably for longer than ten seconds, and requiring them to interact with them can increase brand recollection, click ratios, and can positively influence the user experience. The option to remove an unwanted ad also greatly influences the user experience, and that offering the user a method to ensure that ad takes no excess screen space is a great quality.

\section{ACKNOWLEDGEMENTS}

Acknowledgement: This work is partially funded by the Academy of Finland (Grants 276786-AWARE, 286386CPDSS, 285459-iSCIENCE, 304925-CARE), the European Commission (Grant 6AIKA-A71143-AKAI), and Marie Skłodowska-Curie Actions (645706-GRAGE).

\section{REFERENCES}

1. Mirriad Advertising. 2016. ORC International Survey.

2. Yakov Bart, Andrew T Stephen and Miklos Sarvary. 2014. Which products are best suited to mobile advertising? A field study of mobile display advertising effects on consumer attitudes and intentions. Journal of Marketing Research, 51 (3). 270-285.

3. Jan Panero Benway. Year. Banner blindness: The irony of attention grabbing on the World Wide Web. In Proceedings of the Human Factors and Ergonomics Society Annual Meeting, SAGE Publications Sage CA: Los Angeles, CA, 463-467.

4. Moira Burke, Anthony Hornof, Erik Nilsen and Nicholas Gorman. 2005. High-cost banner blindness: Ads increase perceived workload, hinder visual search, and are forgotten. ACM Transactions on ComputerHuman Interaction (TOCHI), 12 (4). 423-445.

5. Raymond Chen. 2003. The default answer to every dialog box is "Cancel", Microsoft.

6. Chang-Hoan Cho. 2003. Factors influencing clicking of banner ads on the WWW. CyberPsychology \& Behavior, 6 (2). 201-215.

7. Kushal S Dave and Vasudeva Varma. Year. Learning the click-through rate for rare/new ads from similar ads. In Proceedings of the 33rd international ACM SIGIR conference on Research and development in information retrieval, ACM, 897-898.

8. Fd D. Davis. 1989. Perceived Usefulness, Perceived Ease Of Use, And User Acceptance Of Information Technology. MIS quarterly.

9. Subhankar Dhar and Upkar Varshney. 2011. Challenges and business models for mobile locationbased services and advertising. Communications of the ACM, 54 (5). 121-128.
10. D. Duncan. 2004. Method and system for mobile display of context-based advertising content, Google Patents.

11. GroupM. 2016. This Year Next Year: Worldwide Media and Marketing Forecasts, GroupM, 44.

12. Ailsa Kolsaker and Nikolaos Drakatos. 2009. Mobile advertising: The influence of emotional attachment to mobile devices on consumer receptiveness. Journal of Marketing Communications, 15 (4). 267-280.

13. Prestige Marketing. 2015. Who Looks at Banner Ads.

14. José Martí Parreño, Silvia Sanz-Blas, Carla Ruiz-Mafé and Joaquin Aldás-Manzano. 2013. Key factors of teenagers' mobile advertising acceptance. Industrial Management \& Data Systems, 113 (5). 732-749.

15. Suman Nath, Felix Xiaozhu Lin, Lenin Ravindranath and Jitendra Padhye. Year. SmartAds: bringing contextual ads to mobile apps. In Proceeding of the 11th annual international conference on Mobile systems, applications, and services, ACM, 111-124.

16. William J Stanton, Michael J Etzel and Bruce J Walker. 1994. Fundamentals of marketing. McGrawHill College.

17. Melody M Tsang, Shu-Chun Ho and Ting-Peng Liang. 2004. Consumer attitudes toward mobile advertising: An empirical study. International journal of electronic commerce, 8 (3). 65-78.

18. Choy-Har Wong, Garry Wei-Han Tan, Boon-In Tan and Keng-Boon Ooi. 2015. Mobile advertising: the changing landscape of the advertising industry. Telematics and Informatics, 32 (4). 720-734.

19. Byunghwa Yang, Youngchan Kim and Changjo Yoo. 2013. The integrated mobile advertising model: The effects of technology-and emotion-based evaluations. Journal of Business Research, 66 (9). 1345-1352. 